\title{
10. SOBRE LA PALINOLOGÍA DE ALGUNAS ESPECIES ENDÉMICAS E INTERESANTES DE ANDALUCÍA ORIENTAL. V
}

\author{
M. Mar TRIGO, Marta RECIO y Baltasar CABEZUDO
}

Palabras clave. Palinología, Andalucía, España.

Continuando con la serie ya iniciada (Trigo y Salvo, 1988; Bootello et al., 1989; Trigo et al., 1990, 1991.), en el presente trabajo se estudian con el microscopio óptico (MO) y con el microscopio electrónico de barrido (MEB), la morfología polínica de 13 especies de carácter endémico o distribución muy restringida en Andalucía Oriental.

De la mayoría de estos táxones no tenemos referencias de que hayan sido estudiados anteriormente desde el punto de vista palinológico, si bien algunos de ellos ya habían sido incluidos en trabajos más amplios, como es el caso de Zygophyllumfabago (Ubera et al., 1984), Cneorum tricoccon (Lobreau-Callen \& Jeremie, 1986), Buxus balearica (Mateu et al., 1987), Convolvulus boissieri (Polo y Díez, 1985, 1987) у Rosmarinus tomentosus (Rosúa-Campos, 1987).

El análisis polínico se ha realizado utilizando material de herbario del Departamento de Biología Vegetal de la Facultad de Ciencias de la Universidad de Málaga (MGC) y siguiendo los mismos criterios metodológicos y nomenclaturales que en los trabajos anteriores de esta serie. En algún caso, se han agrupado varias especies en base a la afinidad morfológica que presentan sus granos de polen.

\section{RESULTADOS}

Quercus alpestris Boiss. (Fagaceae)

Polen trizonocolporado (colporoidado). Isopolar, radiosimétrico. Circular en v.p. y c.o.e., de subcircular a ligeramente elíptico en v.e. y c.o.m., de suboblado a subprolado, siendo $\mathrm{P} / \mathrm{E}=0,86-1,28(1,03 \pm 0,08)$. Tamaño de pequeño a mediano, con valores de $\mathrm{P}=24-29(26,62 \pm 1,32) \mu \mathrm{m}$ y de $\mathrm{E}=21-28(25,92 \pm 1,36) \mu \mathrm{m}$. Aberturas compuestas; ectoaberturas de tipo colpo, terminales, con la membrana apertural granulosa; endoaberturas de tipo poro, lolongadas y bastante difusas. Exina de aproximadamente $1,5 \mu \mathrm{m}$ de grosor, con la sexina ligeramente más gruesa que la nexina, formando esta última costillas a nivel de las endoaberturas. Téctum completo. Infratéctum con columelas poco patentes. Superficie granulosa, con los gránulos de hasta $1 \mu \mathrm{m}$ de diámetro irregularmente distribuidos por toda la superficie del grano de polen. (Lám. I, fotos 1 a 6).

\section{Succowia balearica (L.) Medicus (Brassicaceae)}

Polen trizonocolpado. Isopolar, radiosimétrico. De circular a subtriangular planaperturado en v.p. y c.o.e., de circular a ligeramente elíptico en v.e. y c.o.m., de suboblado a prolado-esferoidal, siendo $\mathrm{P} / \mathrm{E}=0,83-1,08(0,99 \pm 0,05)$. Tamaño de pequeño a mediano, con valores de $\mathrm{P}=20-27(24,71 \pm 1,42) \mu \mathrm{m}$ y de $\mathrm{E}=24-25(24,76$ $\pm 0,38) \mu \mathrm{m}$. Aberturas simples, de tipo colpo, terminales, con la membrana apertural gemulosa. Exina de 2-2,5 $\mu \mathrm{m}$ de grosor, con la sexina ligeramente más gruesa que la nexina. Téctum parcial. Infratéctum columelado, con columelas gruesas. Superficie reticulada, con lúmenes de contorno ondulado de hasta $2 \mu \mathrm{m}$ de longitud y muros crestados. En el interior de los lúmenes a veces se observan algunas columelas libres. (Lám. I, fotos 7 a 12). 


\section{Zygophyllum fabago L. (Zygophyllaceae)}

Polen trizonocolporado. Isopolar, radiosimétrico. Circular lobulado en v.p. y c.o.e., elíptico en v.e. y c.o.m., de prolado-esferoidal a prolado, siendo $\mathrm{P} / \mathrm{E}=1,07-1,50$ $(1,18-0,11)$. Tamaño pequeño, con valores de $\mathrm{P}=11-15,5(12,17 \pm 0,80) \mu \mathrm{m}$ y de $\mathrm{E}=$ 8 -11 $(10,24 \pm 0,59) \mu \mathrm{m}$. Aberturas compuestas; ectoaberturas de tipo colpo, terminales, con la membrana apertural rugosa; endoaberturas de tipo poro, más o menos circulares, a veces lolongadas. Exina de aproximadamente $1 \mu \mathrm{m}$ de grosor, con la sexina más gruesa que la nexina, formando esta última costillas a nivel de las endoaberturas. Téctum parcial. Infratéctum columelado. Superficie reticulada, con lúmenes irregulares de hasta $1 \mu \mathrm{m}$ de longitud, haciéndose perforada en las zonas polares y en las proximidades de los colpos; muros psilados. (Lám. I, fotos 13 a 16).

\section{Cneorum tricoccon L. (Cneoraceae)}

Polen trizonocolporado. Isopolar, radiosimétrico. Circular, a veces lobulado, en v.p. y c.o.e., fosaperturado; elíptico en v.e. y c.o.m., de prolado-esferoidal a prolado, siendo $\mathrm{P} / \mathrm{E}=1,06-1,52(1,30 \pm 0,13)$. Tamaño de mediano a grande, con valores de $\mathrm{P}=40-51(45,3 \pm 2,60) \mu \mathrm{m}$ y de $\mathrm{E}=29-45(34,87 \pm 3,10) \mu \mathrm{m}$. Aberturas compuestas; ectoaberturas de tipo colpo, terminales, con la membrana apertural granulosa; endoaberturas de tipo colpo, lolongadas o, a veces, más o menos circulares. Exina de 3-4 $\mu \mathrm{m}$ de grosor, con la sexina unas 3 veces más gruesa que la nexina, formando esta última costillas a nivel de las endoaberturas. Téctum parcial. Infratéctum columelado. Superficie estriado-reticulada, con los elementos dispuestos paralelamente al eje polar.(Lám. II, fotos 1 a 8).

\section{Buxus balearica Lam. (Buxaceae)}

Polen polipantoporado. Apolar, con simetría radial. Circular, a veces ligeramente elíptico, en corte óptico. Esferoidal. Tamaño mediano, siendo D= 28-34 (31 $\pm 1,5) \mu \mathrm{m}$. Aberturas simples, de tipo poro y contorno irregular, dispuestas en número de 26 a 45 por grano de polen, a veces difusos cuando se observan con el microscopio óptico; membrana apertural granulosa. Exina de aproximadamente $2 \mu \mathrm{m}$ de grosor, con la sexina ligeramente más gruesa que la nexina. Téctum parcial. Infratéctum columelado. Superficie perforado-reticulada, con lúmenes irregulares de hasta $1 \mu \mathrm{m}$ de longitud, a menudo anastomosados; muros ondulados. Como elementos supratectales aparecen espínulas de $0,1 \mu \mathrm{m}$ de altura. (Lám. III, fotos 1 a 4).

\section{Convolvulus boissieri Steudel (Convolvulaceae)}

Polen trizonocolpado. Isopolar, radiosimétrico. Circular en v.p. y c.o.e., de subcircular a elíptico en v.e. y c.o.m., de suboblado a subprolado, siendo $\mathrm{P} / \mathrm{E}=0,79$ $1,18(1 \pm 0,09)$. Tamaño de mediano a grande, con valores de $\mathrm{P}=44-63(54,06 \pm 4,45)$ $\mu \mathrm{m}$ y de $\mathrm{E}=49-61(53,82 \pm 3,28) \mu \mathrm{m}$. Aberturas simples, de tipo colpo, terminales, con la membrana apertural densamente cubierta de gemas y gránulos de superficie espinulosa. Exina de 3-3,5 $\mu \mathrm{m}$ de grosor, con la sexina 2 veces más gruesa que la nexina. Téctum perforado. Infratéctum columelado. Superficie perforada, con perforaciones más o menos circulares, apareciendo espínulas de hasta $0,3 \mu \mathrm{m}$ de altura como elementos supratectales. (Lám. II, fotos 9 a 16).

Nepeta amenthystina Poiret subsp. anticaria Cabezudo, Nieto Caldera et Navarro (Lamiaceae)

Rosmarinus tomentosus Hubert-Morath et Maire (Lamiaceae)

Polen hexazonocolpado. Isopolar, con simetría radial. Circular, a veces elíptico en v.p. y c.o.e., de subcircular a ligeramente elíptico en v.e. y c.o.m., de suboblado a subprolado, siendo $\mathrm{P} / \mathrm{E}=0,90-1,29(1,04 \pm 0,11)$ en $N$. amenthystina y 0,77-1,33 (1,03 
$\pm 0,15)$ en $R$. tomentosus. Tamaño mediano, con valores de $\mathrm{P}=26-31(28,63 \pm 1,69)$ $\mu \mathrm{m}$ en $N$. amenthystina y $27-36(32,16 \pm 2,50) \mu \mathrm{m}$ en $R$. tomentosus y de $\mathrm{E}=24-30$ $(27,63 \pm 1,63) \mu \mathrm{m}$ en $N$. amenthystina y $26,5-36(31,28 \pm 2,76) \mu \mathrm{m}$ en $R$. tomentosus. Aberturas simples, de tipo colpo, terminales, con la membrana apertural densamente granulosa. Exina de 1,5-2 $\mu \mathrm{m}$ de grosor, con la sexina 1,5 veces más gruesa que la nexina. Téctum parcial. Infratéctum columelado. Superficie doblemente reticulada, con un retículo de primario (escasamente marcado en $N$. amenthystina) de lúmenes irregulares en el interior de los cuales aparece otro con lúmenes más pequeños de hasta 0,5 $\mu \mathrm{m}$ de diámetro; muros psilados. (Lám. III, fotos 5 a 12).

\section{Lafuentea rotundifolia Lag.(Scrophulariaceae)}

Polen trizonocolporado (colporoidado). Isopolar, radiosimétrico. Circular en v.p. y c.o.e., de subcircular a ligeramente elíptico en v.e. y c.o.m., de oblado-esferoidal a prolado, siendo $\mathrm{P} / \mathrm{E}=0,97-1,42(1,18 \pm 0,11)$. Tamaño pequeño, con valores de $\mathrm{P}=$ $13,5-17,5(15,41 \pm 1,06) \mu \mathrm{m}$ y de $\mathrm{E}=11,5-15,5(13,06 \pm 0,91) \mu \mathrm{m}$. Aberturas compuestas; ectoaberturas de tipo colpo, terminales, constreñidas en la zona ecuatorial, con la membrana apertural psilada; endoaberturas de tipo poro, bastante difusas. Exina de aproximadamente $1 \mu \mathrm{m}$ de grosor, con la sexina de 2 a 3 veces más gruesa que la nexina, formando esta última costillas a nivel de las endoaberturas. Téctum parcial. Infratéctum columelado. Superficie finamente reticulada, con lúmenes irregulares de hasta $1 \mu \mathrm{m}$ de longitud, haciéndose perforada en las proximidades de los colpos; muros psilados. (Lám. III, fotos 13 a 16).

\section{Anacyclus alboranensis Esteve (Asteraceae)}

Polen trizonocolporado. Isopolar, radiosimétrico. Circular en v.p. y c.o.e., de circular a subcircular en v.e. y c.o.m., de oblado-esferoidal a prolado-esferoidal, siendo $\mathrm{P} / \mathrm{E}=0,96-1,08(1 \pm 0,03)$. Tamaño de pequeño a mediano, con valores de $\mathrm{P}=$ 24-27 $(25,24 \pm 1,01) \mu \mathrm{m}$ y de $\mathrm{E}=24-27(25,08 \pm 0,86) \mu \mathrm{m}$. Aberturas compuestas; ectoaberturas de tipo colpo, subterminales, con la membrana apertural granulosa; endoaberturas de tipo poro, más o menos circulares o elípticas, lalongadas. Exina de 3,5 a $4,5 \mu \mathrm{m}$ de grosor, con la sexina de 2 a 2,5 veces más gruesa que la nexina, engrosándose ésta última a nivel de las endoaberturas para formar costillas. Téctum perforado, con columelas intratectales que forman elevaciones del mismo. Infratéctum columelado. Superficie perforada, con espínulas de hasta $2 \mu \mathrm{m}$ de altura regularmente dispuestas sobre las elevaciones del téctum. (Lám. IV, fotos 1 a 7).

\section{Senecio malacitanus Huter subsp. malacitanus (Asteraceae)}

Polen trizonocolporado. Isopolar, con simetría radial. De contorno circular en v.p. y c.o.e., sinuaperturado; de subcircular a ligeramente elíptico en v.e. y c.o.m., de oblado-esferoidal a subprolado, siendo $\mathrm{P} / \mathrm{E}=0,91-1,25(1 \pm 0,04)$. Tamaño de pequeño a mediano, con valores de $\mathrm{P}=21-27(25,25 \pm 1,33) \mu \mathrm{m}$ y de $\mathrm{E}=23-26(25,02$ $\pm 0,92) \mu \mathrm{m}$. Aberturas compuestas; ectoaberturas de tipo colpo, subterminales, con la membrana apertural granulosa; mesoaberturas y endoaberturas de tipo poro, lalongadas. Exina de 3-3,5 $\mu \mathrm{m}$ de grosor, con la sexina 2 veces más gruesa que la nexina, formando esta última costillas a nivel de las endoaberturas. Téctum perforado, con columelas intratectales. Infratéctum columelado. Superficie de perforada a finamente reticulada con espínulas de hasta $2 \mu \mathrm{m}$ de longitud regularmente distribuidas y dispuestas sobre elevaciones del téctum. (Lám. IV, fotos 8 a 14).

\section{Centaurea lainzii Fernández Casas (Asteraceae)}

Polen trizonocolporado. Isopolar, radiosimétrico; a veces hexazonocolporado y heteropolar (3+3). Circular lobulado en v.p. y c.o.e., fosaperturado; elíptico en v.e. 
y c.o.m., de prolado-esferoidal a prolado, siendo $\mathrm{P} / \mathrm{E}=1,12-1,45(1,25 \pm 0,08)$. Tamaño grande, con valores de $\mathrm{P}=53-64(58 \pm 2,93) \mu \mathrm{m}$ y de $\mathrm{E}=41-53(46,4 \pm 3,43)$ $\mu \mathrm{m}$. Aberturas compuestas; ectoaberturas de tipo colpo, terminales; mesoaberturas lolongadas y endoaberturas lalongadas, a veces formando endocíngulo. Exina de 8 a $10 \mu \mathrm{m}$ de grosor, con la sexina de 1,5 a 2 veces más gruesa que la nexina, formando esta última costillas a nivel de las endoaberturas. Téctum completo, con columelas intratectales. Infratéctum sin columelas aparentes, siendo frecuente la aparición de cáveas entre sexina y nexina que a menudo llegan a separar totalmente a estas dos capas. Superficie microperforada, con espínulas cónicas de hasta $1,5 \mu \mathrm{m}$ de altura uniformemente distribuidas por toda la superficie del grano de polen. (Lám. V, fotos 1 a 7$)$.

En las muestras estudiadas aparecen gran cantidad de granos de polen aberrantes y con número de aberturas anómalo.

\section{Hieracium texedense Pau (Asteraceae)}

Polen trizonocolporado. Isopolar, con simetría radial. Hexagonal en v.p. y c.o.e., subcircular en v.e. y c.o.m., oblado-esferoidal, siendo $\mathrm{P} / \mathrm{E}=0,91-1(0,93 \pm$ $0,02)$. Tamaño mediano, con valores de $\mathrm{P}=30-32(31 \pm 1,41) \mu \mathrm{m}$ y de $\mathrm{E}=32-35(33$ $\pm 1,42) \mu \mathrm{m}$. Aberturas compuestas; ectoaberturas de tipo colpo, subterminales, con la membrana apertural psilada; mesoaberturas difusas y endoaberturas lolongadas, redondeadas o ligeramente elípticas. Exina de 5,5-6 $\mu \mathrm{m}$ de grosor a nivel de las crestas, con la sexina de 4 a 5 veces más gruesa que la nexina. Téctum parcial. Infratéctum con columelas poco patentes. Superficie equinolofada, con 15 lagunas bien diferenciadas: 6 polares $(3+3), 6$ subpolares $(3+3)$ y 3 ecuatoriales y 2 casquetes polares. Las lagunas se encuentran delimitadas por crestas que al igual que los casquetes polares se encuentran perforadas y ornamentadas con espínulas de 2 a $3 \mu \mathrm{m}$ de altura.(Lám. V, fotos 8 a 13).

\section{DISCUSIÓN}

Tras el estudio realizado hemos podido comprobar que los resultados obtenidos coinciden en general con la bibliografía consultada para aquéllos táxones que ya habían sido tratados anteriormente desde el punto de vista palinológico, encontrándose sólo algunas diferencias en la interpretación de los resultados. Así por ejemplo consideramos las aberturas de Zygophyllum fabago como colporadas mientras que Ubera et al. (1984) lo hacen como colporoidadas. Por otra parte, Polo y Díez (1987) basan la separación de los granos de polen de las distintas especies del género Convolvulus en la presencia de gránulos espinulosos sobre la membrana apertural de los colpos, considerando que C. boissieri posee gránulos lisos. No obstante, en la población que aquí se estudia hemos observado que dichos gránulos son equinulados.

Algunos autores (Luque y Candau, 1987; Rosúa-Campos, 1987) han considerado los granos de polen de Nepeta y Rosmarinus como de simetría bilateral. Sin embargo, creemos que la forma elíptica que a menudo presentan dichos granos de polen cuando se observan con el MO en v.p. y c.o.e. pueden ser debida a deformaciones del material y que dada la posición radial de las aberturas se hace muy difícil establecer con exactitud los ejes $E_{1}$ y $E_{2}$. En cualquier caso, los hemos considerados como radiosimétricos.

Por la comparación con los datos aportados por otros autores (Saenz, 1973; Diez y Suárez, 1987), no se han encontrado diferencias significativas entre la morfología polínica de Quercus alpestris y otras especies afines como $Q$. faginea Lam., Q. canariensis Willd., Q. pyrenaica Willd., Q. fruticosa Brot. o $Q$. suber L., por lo que parece ser que a estos niveles la morfología polínica tiene escaso valor taxonómico. 


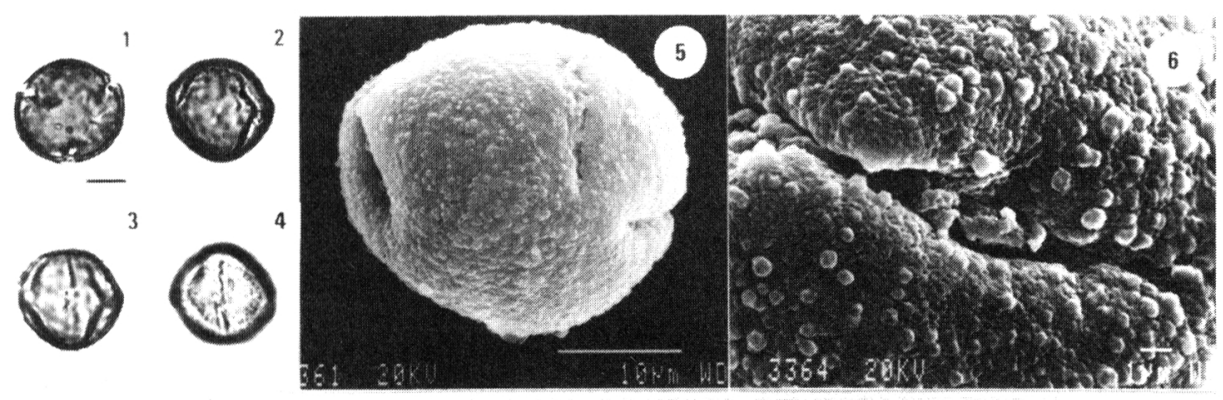

7

8

9

10
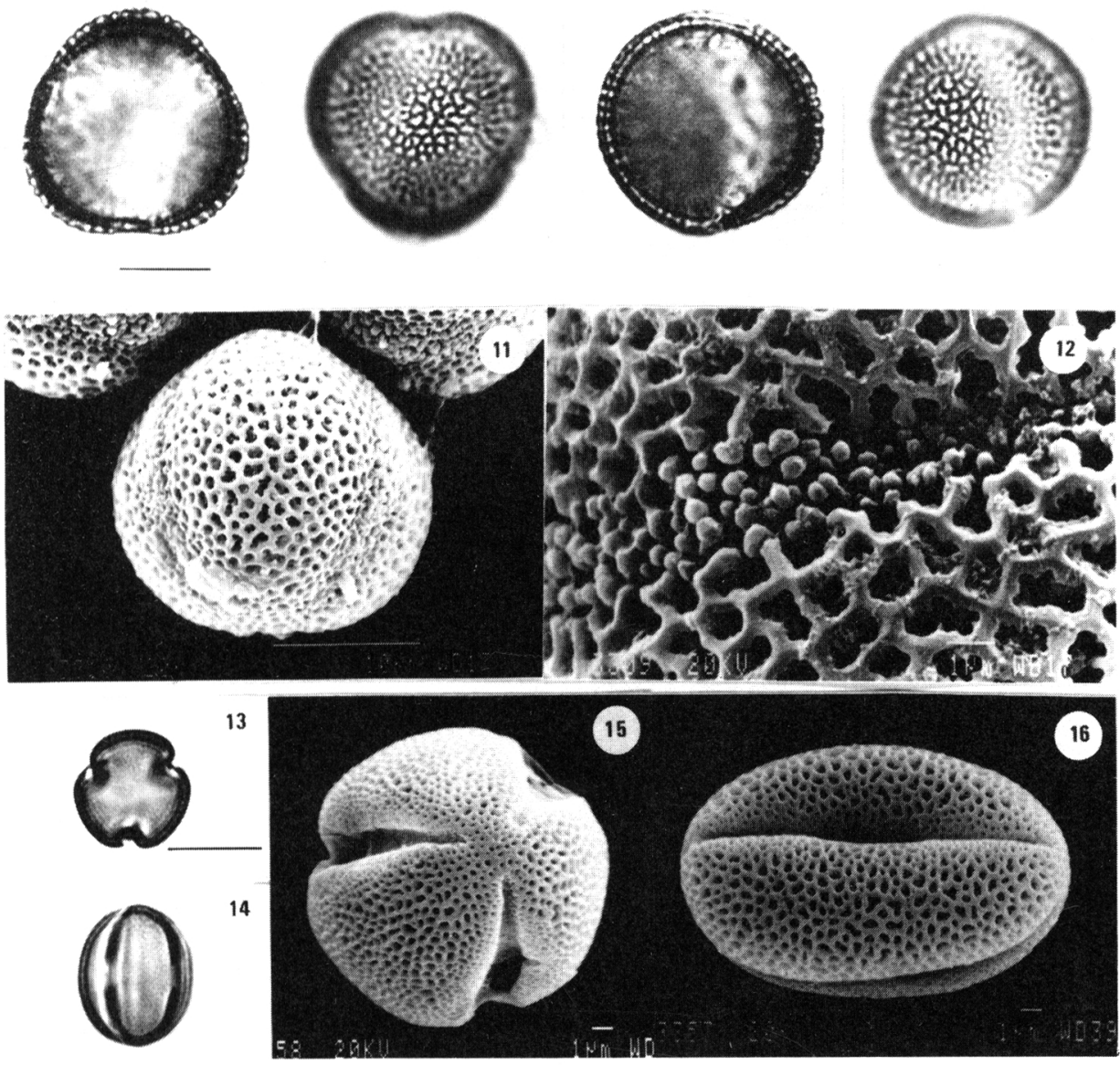

Lámina I: Quercus alpestris: 1) v.p. y c.o.e. 2) v.e. y c.o.m. 3) v.e., costillas. 4) v.e., abertura. 5) aspecto general del polen. 6) detalle de la ornamentación y abertura. Sucowia balearica: 7) v.p. y c.o.e. 8) v.p., superficie. 9) v.e. y c.o.m. 10) v.e., superficie. 11) aspecto general del polen. 12) detalle de la ornamentación y abertura. Zygophyllum fabago: 13) v.p. y c.o.e. 14) v.e. y c.o.m. 15) aspecto general del polen, v.p. 16) aspecto general del polen, v.e. Escala M.O.: $10 \mu \mathrm{m}$. 

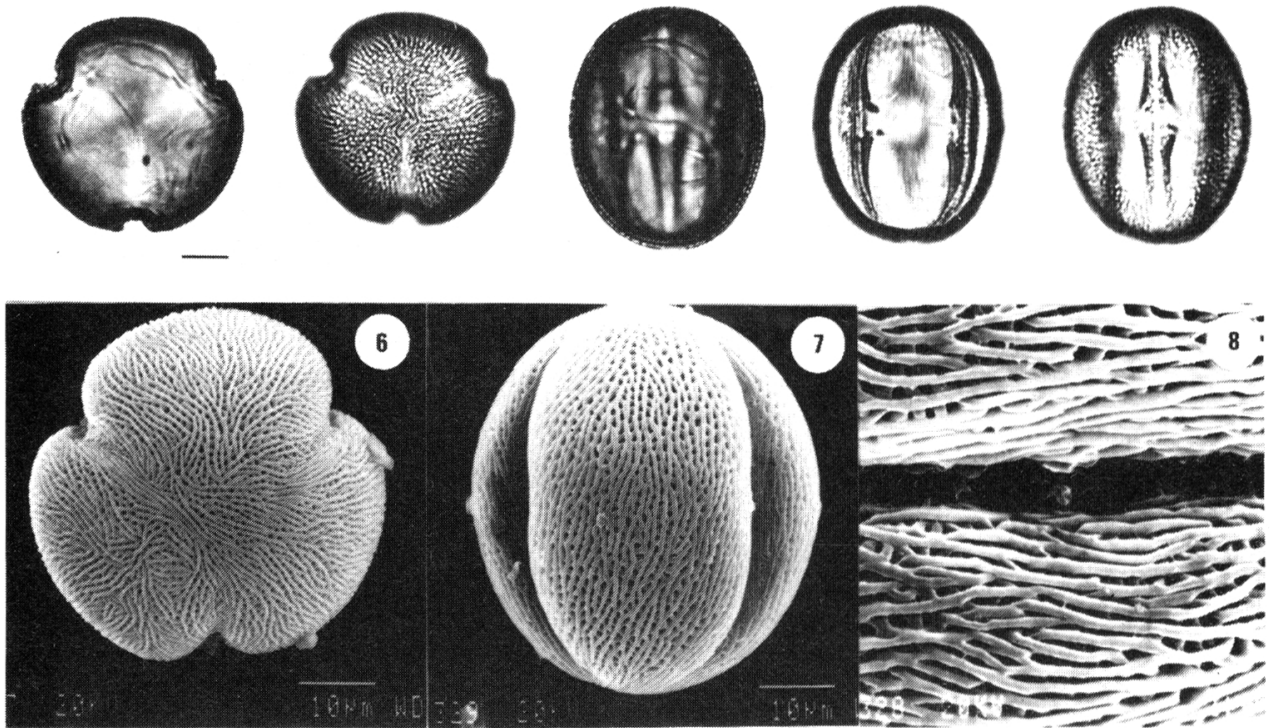

9

10

11

12
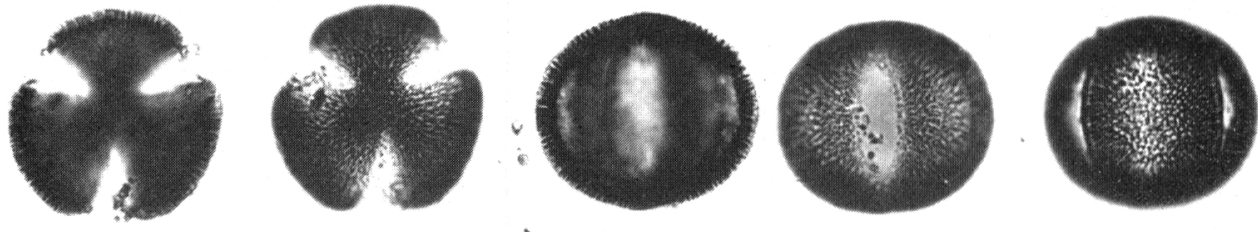

$\checkmark$
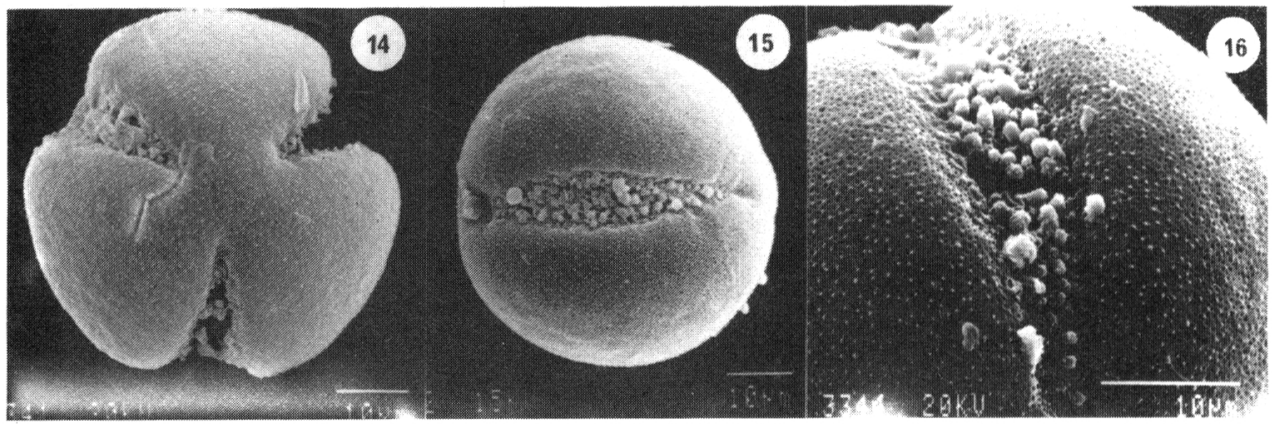

Lámina II: Cneorum tricoccon: 1) v.p. y c.o.e. 2) v.p., superficie. 3) v.e. y c.o.m. 4) v.e., costillas. 5) v.e., abertura. 6) aspecto general del polen, v.p. 7) aspecto general del polen, v.e. 8) detalle de la superficie y abertura. Convolvulus boissieri: 9) v.p. y c.o.e. 10) v.p., superficie. 11) v.e. y c.o.m. 12) v.e., abertura. 13) v.e., superficie. 14) aspecto general del polen, v.p. 15) aspecto general del polen, v.e. 16) detalle de la superficie y abertura. Escala M.O.: $10 \mu \mathrm{m}$. 

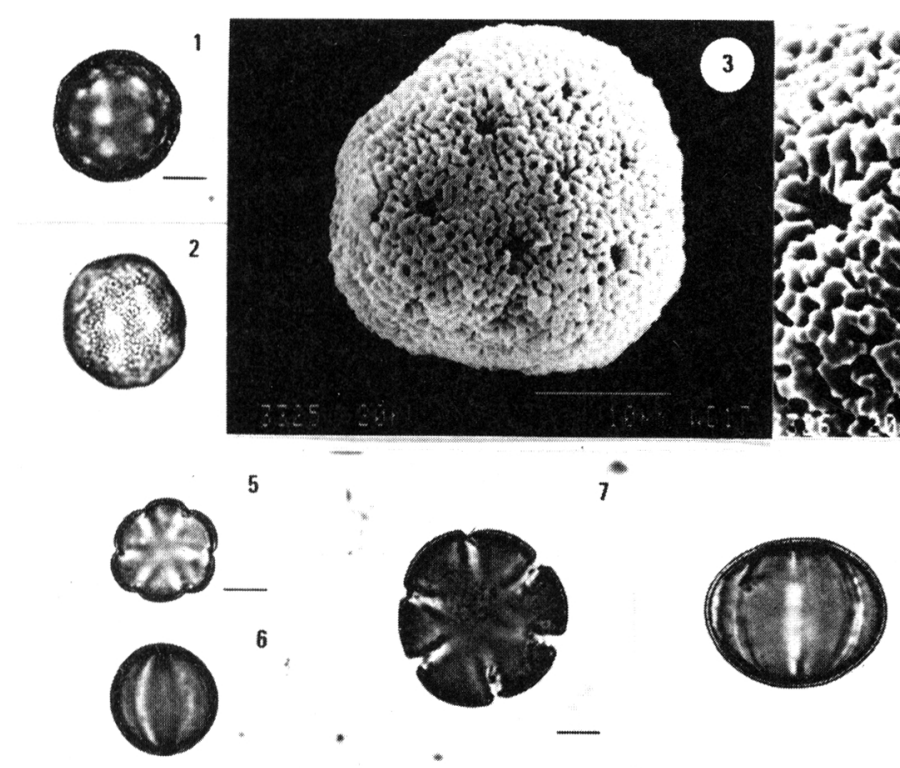

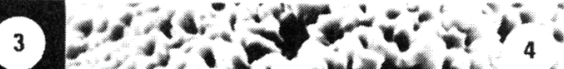

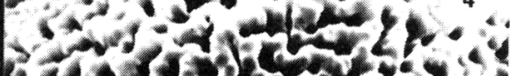

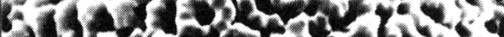

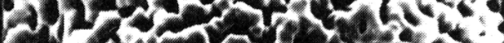
- sustarosis.

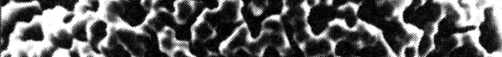
30. 4...

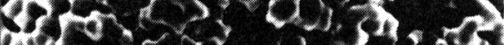

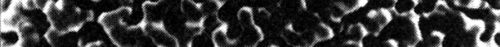

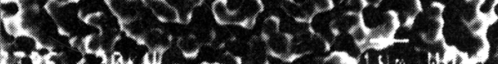

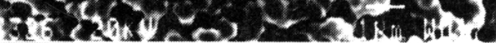

9
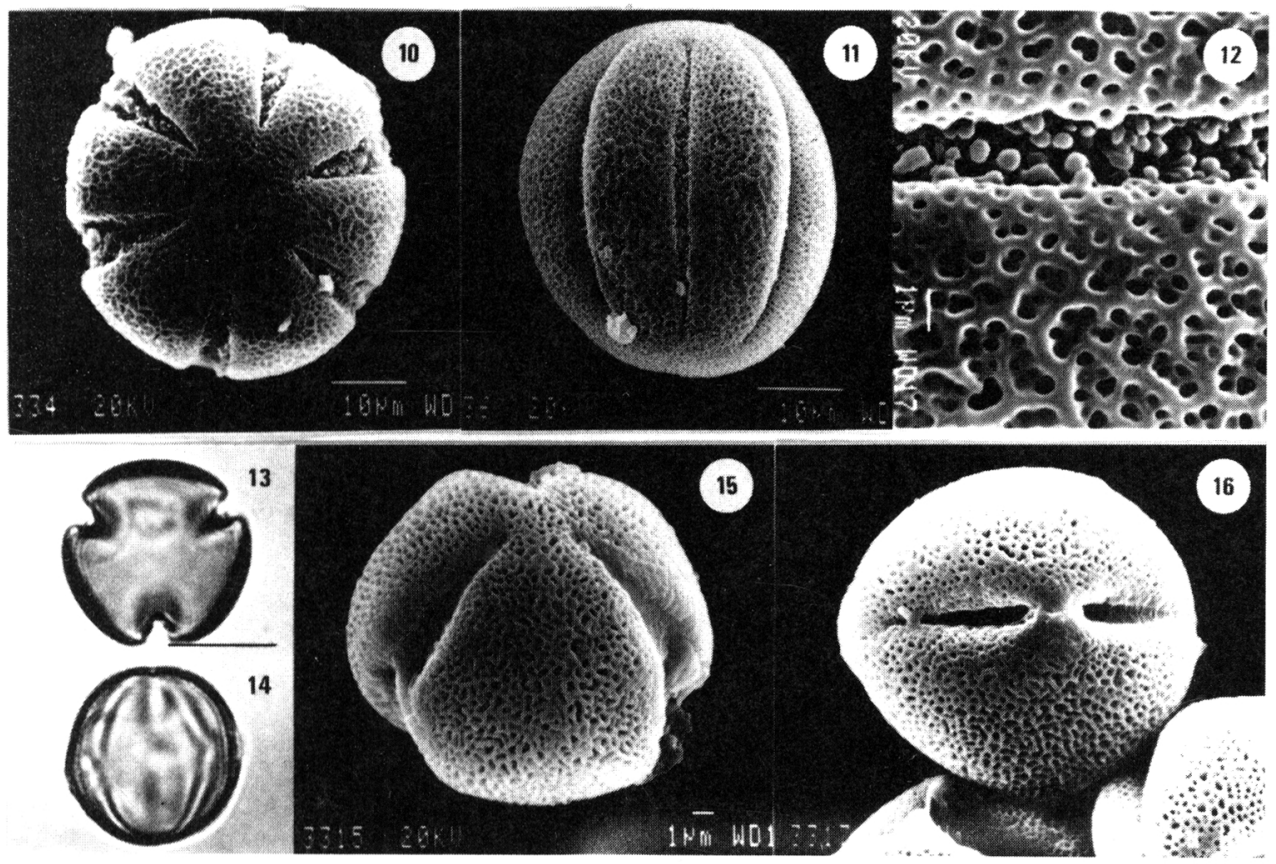

Lámina III: Buxus balearica: 1) corte óptico. 2) superficic. 3) aspecto general del polen. 4) detalle de la superficie y aberturas. Nepeta amenthystina: 5) v.p. y c.o.e. 6) v.e. y c.o.m. Rosmarinus tomentosus: 7) v.p. y c.o.e. 8) v.e. y c.o.m. 9) v.e., superficie y aberturas. 10) aspecto general del polen, v.p. 11) aspecto general del polen, v.e. 12) detalle de la superficie y abertura. Lafuentea rotundifolia: 13) v.p. y c.o.e., 14) v.e. y c.o.m. 15) aspecto general del polen, v.p. 16) aspecto general del polen, v.e. Escala M.O.: $10 \mu \mathrm{m}$. 


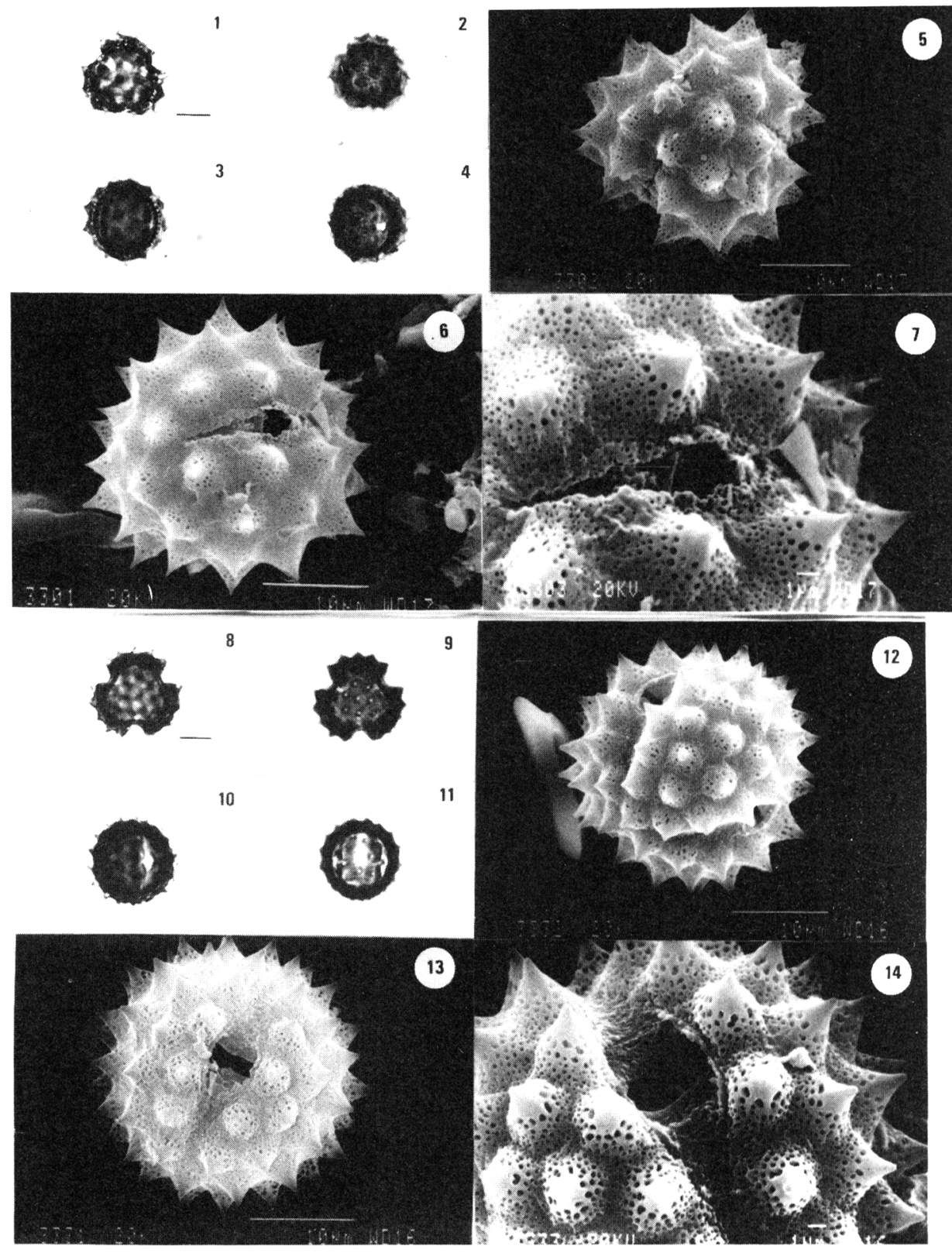

Lámina IV: Anacyclus alboranensis: 1) v.p. y c.o.e. 2) v.p., superficie. 3) v.e. y c.o.m. 4) v.e., superficie. 5) aspecto general del polen, v.p. 6) aspecto general del polen, v.e. 7) detalle de la superficie y abertura. Senecio malacitanus: 8) v.p. y c.o.e. 9) v.p., superficie. 10) v.e. y c.o.m. 11) v.e., costillas. 12) y 13) aspecto general del polen, v.e. 14) detalle de la superficie y abertura. Escala M.O.: $10 \mu \mathrm{m}$. 


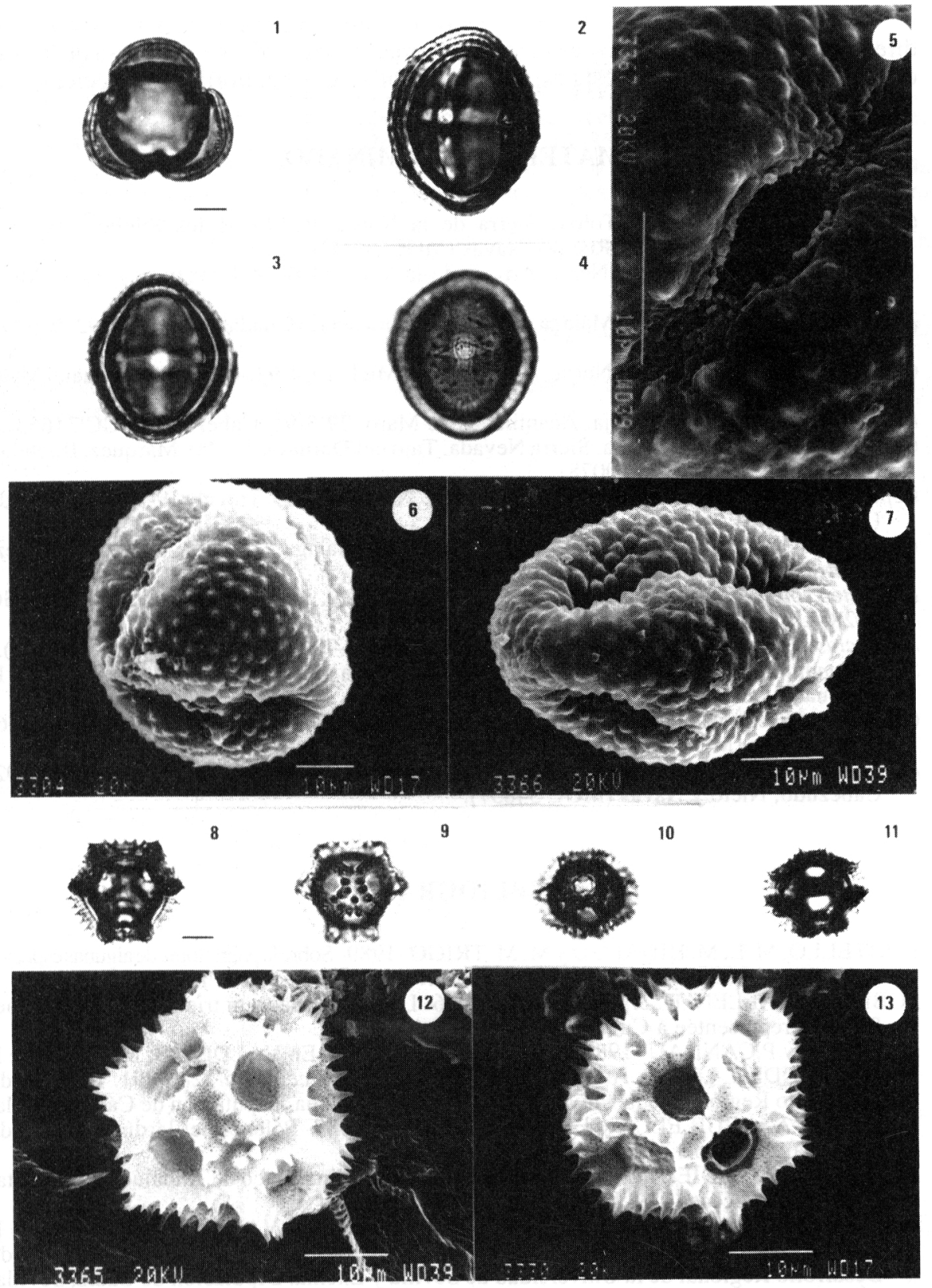

Lámina V: Centaurea lainzii: 1) v.p. y c.o.e. 2) v.e. y c.o.m. 3) v.e., costillas. 4) v.e., abertura. 5) detalle de la superficie y abertura. 6) aspecto general de un grano de polen hexacolpado, v.p.. 7) aspecto general de un grano de polen tricolporado, v.e. Hieracium texedense: 8)v.p. y c.o.e. 9) v.p., superficie. 10) v.e., abertura. 11) v.e. y c.o.m. 12) aspecto general del polen, v.p. 13) aspecto general del polen, v.e. Escala M.O.: $10 \mu \mathrm{m}$. 
Centurea lainzii presenta un elevado porcentaje de granos de polen aberrantes, diferentes tamaños y número de aberturas anómalos, que podría ser la causa de la falta de reproducción sexual en esta especie y apoyar su orígen hibridógeno reciente.

\section{MATERIAL EXAMINADO.}

Quercus alpestris: Málaga. Tolox. Sierra de la Nieve. Puerto de los Pilones. 28-5-92. Cabezudo, Pérez-Latorre, Recio y Navas (MGC 34243).

Succowia balearica: Málaga. Nerja. Arroyo de la Miel. 14-4-92. Cabezudo y Suau (MGC 34290).

Zygophyllumfabago: Málaga. Málaga. Desembocadura del rio Guadalhorce, 26-5-92. Esteban (MGC 17459).

Cneorum tricoccon: Málaga. Nerja. Arroyo de la Miel. 14-4-92. Cabezudo y Suau (MGC 34289).

Buxus balearica: Málaga. Nerja. Acantilados de Maro. 29-3-91. Cabezudo (MGC 31653).

Convolvulus boissieri: Granada. Sierra Nevada. Tajo del Dornajo. 4-7-90. Márquez, Bootello, Moreno y Navas (MGC 30078).

Nepeta amenthystina: subsp. anticaria. Málaga. Antequera. El Torcal. 19-5-86. Cabezudo (MGC 21348).

Rosmarinus tomentosus: Málaga. Nerja. Puntilla de Málaga. 27-5-92. Cabezudo, PérezLatorre, Recio y Navas (MGC 34248).

Lafuentea rotundifolia: Granada. Otívar. Cruce con Lentejí. 14-5-92 Cabezudo y Suau (MGC 34288).

Anacyclus alboranensis: Granada. Isla de Alborán. 15-4-70. Esteve Chueca (MA 413357).

Senecio malacitanus: Málaga. Montes de Málaga. Subida por el Pantano del Agujero. 28-1190. Suau y col. (MGC 30068).

Centaurea lainzii: Málaga. Estepona. Sierra Bermeja. Subida al Puerto de Peñas Blancas. 66-91. Gálvez (MGC 34286).

Hieracium texedense: Granada. Alhama de Granada. Sierra Tejeda. Salto del Caballo. 9-7-92. Cabezudo, Nieto y Navas (MGC 34857).

\section{BIBLIOGRAFÍA}

BOOTELLO, M. L; M. HIDALGO y M. M. TRIGO - 1989- Sobrelapalinología de algunasespecies endémicas e interesantes de Andalucía oriental II. Acta Bot. Malacitana., 14: 245-253.

LOBREAU-CALLEN, D. \& J. JEREMIE -1986- L'espèce Cneorum tricoccon (Cneoraceae, Rutales) représentée á Cuba. Grana,25: 155-158.

LUQUE, T. y P.CANDAU -1987-. Lamiaceae (Labiateae). En VALDÉS, B; M. J. DÍEZ e I. FERNÁNDEZ, I.(eds.). Atlas polínico de Andalucía Occidental.:283-291. Instituto de Desarrollo Regional de la Universidad de Sevilla y Excma. Diputación de Cádiz, Sevilla.

MATEU, I., A. AGUILELLA y J. M. AZCARRAGA -1987- Pólenes del Jardín Botánico de Valencia. An. Asoc. Palinol. Leng. Esp., 3: 73-90.

POLO, J.M. y M. J. DÍEZ -1985- Contribución al atlas palinológico de Andalucía Occidental II. Convolvulaceae (s.1.). Lagascalia, 13: 239-254.

POLO, J. M. y M. J. DÍEZ -1987- Convolvulaceae. En B. VALDÉS, M. J. DÍEZ E I. FERNÁNDEZ (eds.). Atlas polínico de Andalucía Occidental: 261-264 Instituto de Desarrollo Regional de la Universidad de Sevilla y Excma. Diputación de Cádiz, Sevilla.

ROSÚA-CAMPOS, J. L. -1987- Estudio palinológico del género Rosmarinus L. (Labiatae). An. Asoc. Palinol. Leng. Esp., 3: 13-17 (1987).

SAENZ, C. -1973- Estudios palinológicos sobre Quercus de la España Mediterránea. Bol. $R$. Soc. Española Hist. Nat. (Biol.), 71: 315-329.

SUAREZ, R. y M. J. DÍEZ -1987- Fagaceae. En B. VALDÉS, M. J. DÍEZ E I. FERNÁNDEZ (eds.). Atlas polínico de Andalucía Occidental: 94-96 Instituto de Desarrollo Regional de 
la Universidad de Sevilla y Excma. Diputación de Cádiz, Sevilla.

TRIGO, M. M. y A. E. SALVO -1988- Sobre la palinología de algunas especies endémicas e interesantes de Andalucía oriental. I. Acta Bot. Malacitana, 13: 347-353.

TRIGO, M. M; M. I. HIDALGO, I. GARCÍA, M. L. BOOTELLO, J. M. NIETO CALDERA y B.CABEZUDO -1990-Sobre la palinología de algunas especies endémicas e interesantes de Andalucía Oriental. III. Acta Bot. Malacitana., 15: 353-365.

TRIGO, M. M; M. I. HIDALGO, M. L. BOOTELLO y B. CABEZUDO -1991- Sobre la palinología de algunas especies endémicas e interesantes de Andalucía oriental. IV. Acta Bot. Malacitana., 16: 500-508.

UBERA, J. L., C. GALÁN y P. CANDAU -1984- Estudio polínico de Zygophyllaceae L. en la Península Ibérica. An. Asoc. Palinol. Leng. Esp., 1: 39-48.

(Aceptado para su publicación en Junio de 1992)

Dirección de los autores. Departamento de Biología Vegetal Facultad de Ciencias. Universidad de Málaga. Ap. 59. 29080. Málaga. 